\title{
Retrospective Analysis of Idiopathic Granulomatous Mastitis: Its Diagnosis and Treatment
}

\author{
Jinwoo Jeon, Kyunghee Lee, Yunyeong Kim, Yong Soon Chun, Heung Kyu Park \\ Department of Surgery, Gachon University Gil Medical Center, Incheon, Korea
}

Purpose: Idiopathic granulomatous mastitis (IGM) is a rare disease characterized by noncaseating granulomatous inflammation of unknown origin. Because its clinical features are similar to those of other type of mastitis or breast cancer, accurate diagnosis and adequate treatment are essential to ensuring a short symptom duration and improving the quality of life. Methods: The clinical, radiologic, pathophysiologic, and treatment data for 43 patients diagnosed with IGM at the Breast Cancer Center of Gachon University Gil Medical Center between 2005 and 2016 were retrospectively reviewed. Results: Forty-one patients (95.34\%) were of childbearing age, seven (16.27\%) had a history of lactation, and five (11.62\%) had a history of oral contraceptive use. In terms of radiologic findings, 30 patients (69.77\%) were diagnosed with Breast Imaging-Reporting and Data System category $\geq 4 \mathrm{~A}$ lesions. Corticosteroid therapy was administered to 36 patients (83.72\%); overall, 18 patients (41.86\%) did not require surgery and 25 patients (58.13\%) underwent partial or total mastectomy. Twelve patients (27.90\%) developed recurrence. Conclusion: IGM is a benign disease that can be misdiagnosed as breast cancer because of its similar clinical and radiologic findings. Proper diagnosis and treatment can be difficult, but delays may lead to prolonged pain and cosmetic and socioeconomic problems. Efforts should be aimed at establishing the cause of IGM and developing efficient protocols for its diagnosis and treatment.

Key Words: Breast, Diagnosis, Granulomatous mastitis, Steroids

\section{INTRODUCTION}

Idiopathic granulomatous mastitis (IGM) is a rare type of mastitis that was first described by Kessler and Wolloch [1], and is characterized by chronic, noncaseating granulomatous inflammation of breast lobules. Kessler and Wolloch [1] and Fletcher et al. [2] reported that the most common clinical presentation is a palpable lobular granulomatous inflammation in the breast with or without pain. The size of the mass varies from 0.5 to $9.0 \mathrm{~cm}$; in some cases, nipple retraction or skin fistulas are observed. Moreover, Jorgensen and Nielsen [3] reported axillary lymph node enlargement in 15\% of patients of IGM. All affected patients are typically of childbearing age and have a history of oral contraceptive use $[1,2,4]$. Antibiotics and corticosteroids or surgery have been reported as treatment options.

Despite its benign nature, IGM is often misdiagnosed as breast cancer because of certain clinical and radiological similarities; therefore, a pathologic diagnosis conducted via biopsy is essential. Moreover, IGM

Correspondence: Heung Kyu Park

Department of Surgery, Gachon University Gil Medical Center, 21 Namdongdaero 774beon-gil, Namdong-gu, Incheon 21565, Korea

Tel: +82-32-460-3244, Fax: +82-32-460-3247, E-mail: hgjh@gilhospital.com

Received: Jun 16, 2017 Revised: Oct 30, 2017 Accepted: Dec 19, 2017 is pathologically characterized by chronic and lobular mastitis with idiopathic, noncaseating granuloma, but often, the histologic findings overlap with those of granulomatous mastitis caused by other etiologies such as mycobacterial infections. Therefore, serological and histological examinations are necessary for achieving a differential diagnosis [3]. IGM is a rare disease and its pathogenesis remains controversial; hence, it can be misdiagnosed at a local clinic, and proper treatment may thus be delayed. In this study, we retrospectively reviewed data for 43 patients with IGM who were diagnosed, treated, and monitored between January 2005 and December 2016.

\section{METHODS}

Herein, a review of medical records from Breast Cancer Center of Gachon University Gil Medical Center for the period of January 2005 to December 2016 resulted in the identification of 43 patients with a pathologically confirmed diagnosis of IGM who were treated and followed up.

Patient information such as medical history, social history and clinical symptoms was collected through initial interviews; subsequently, physicians performed a physical examination. Mammography was 
performed using a Selenia (Hologic Inc., Bedford, USA) full field digital mammography unit for women over the age of 30 years, except for those unable to undergo the procedure because of pain or pus drainage. Ultrasonography was performed on all patients by radiologists and the size of the lesion was defined as the major axis length as determined via ultrasonography. At the time of examination, breast lesions were evaluated and enlargement of axillary lymph nodes was confirmed.

To establish a pathophysiologic diagnosis, three to five specimens were collected using via fine needle aspiration (FNA), core needle biopsy (CNB), or both. FNA was performed using a $20 \mathrm{~mL}$ syringe with a 20-gauge needle, and the CNB was performed using an ultrasonogram and an automatic biopsy gun equipped with a 14-gauge needle under lidocaine local anesthesia.

The tissues obtained via FNA or CNB were stained with hematoxylin and eosin, Gomori methenamine silver, and the Gram stain, as well as for acid-fast bacilli, using paraffin-embedded sections. Samples were cultured to exclude infectious causes due to bacteria as well as tubercular bacilli.

\section{RESULTS}

Herein, 41 of the 43 patients (95.34\%) were of childbearing age, ranging from 21 to 50 years, with a median age of 36 years. Of the 43 patients, seven (16.27\%) were breastfeeding prior to the diagnosis of IGM and five (11.62\%) had a history of oral contraceptive use. Six patients (13.95\%) were receiving medication for schizophrenia or bipolar disorder, and two (4.65\%) were receiving medication for diabetes. Thirty-two patients (74.41\%) were diagnosed with simple mastitis and were treated with antibiotics or incisional drainage.

Thirty-two patients (74.41\%) visited Gachon University Gil Medical Center via the local clinic. Physical examinations revealed 21 (48.83\%) cases of IGM of the right breast, and 21 (48.83\%) of the left breast, while one patient had multiple lesions affecting both breasts. Twelve patients (27.90\%) had a body mass index in the normal range $\left(<23 \mathrm{~kg} / \mathrm{m}^{2}\right)$ at the time of diagnosis, nine (20.93\%) were overweight $\left(23-24.9 \mathrm{~kg} / \mathrm{m}^{2}\right)$, and $22(51.16 \%)$ were obese $\left(\geq 25 \mathrm{~kg} / \mathrm{m}^{2}\right)$.

In order to obtain a definite diagnosis, all patients underwent pathological examination using FNA or CNB specimens. Bacteria were detected in two patients; coagulase-negative Streptococcus in one and Streptococcus group C in the other. All specimens were stained for acid fast bacilli to check for the presence of tuberculosis; however, all tests yielded negative results.

All patients received steroid therapy, surgical resection, or both. Patients were monitored on an outpatient basis at intervals of 1 to 2 weeks in the clinic until the lesion disappeared after treatment completion, follow-up visits were scheduled twice at 6-month intervals. Corticosteroids were administered to 36 patients (83.72\%); the other seven patients (16.27\%) underwent surgery immediately after diagnosis. Corticosteroid treatment was initiated at a dose of $0.4 \mathrm{mg} / \mathrm{kg} / \mathrm{day}$. No patient experienced any side effects such as glucose intolerance or

Table 1. Clinical characteristics of patients

\begin{tabular}{|c|c|}
\hline Characteristic & No. of patients (\%) \\
\hline \multicolumn{2}{|l|}{ Age (yr) } \\
\hline$<50$ & $41(95.34)$ \\
\hline$\geq 50$ & $2(4.65)$ \\
\hline \multicolumn{2}{|c|}{ Body mass index $\left(\mathrm{kg} / \mathrm{m}^{2}\right)$} \\
\hline$<23$ & $12(27.90)$ \\
\hline $23-24.9$ & $9(20.93)$ \\
\hline$\geq 25$ & $22(51.16)$ \\
\hline \multicolumn{2}{|c|}{ History of oral contraceptives use } \\
\hline No & $38(88.37)$ \\
\hline Yes & $5(11.62)$ \\
\hline \multicolumn{2}{|l|}{ Lactation } \\
\hline No & $36(83.72)$ \\
\hline Yes & $7(16.27)$ \\
\hline \multicolumn{2}{|l|}{ Location } \\
\hline Right & $21(48.83)$ \\
\hline Left & $21(48.83)$ \\
\hline Both & $1(2.32)$ \\
\hline \multicolumn{2}{|l|}{ Blood culture } \\
\hline None & $41(95.34)$ \\
\hline Done & $2(4.65)$ \\
\hline \multicolumn{2}{|l|}{ Acid fast bacilli stain } \\
\hline Negative & $43(100)$ \\
\hline Positive & 0 \\
\hline \multicolumn{2}{|l|}{ Antibiotics } \\
\hline 2nd cephalosporin & $10(23.25)$ \\
\hline 3rd cephalosporin & $25(58.13)$ \\
\hline Quinolone & $8(18.60)$ \\
\hline \multicolumn{2}{|l|}{ Steroid usage } \\
\hline No & $7(16.27)$ \\
\hline Yes & $36(83.72)$ \\
\hline \multicolumn{2}{|l|}{ Operation } \\
\hline Partial mastectomy & $24(55.81)$ \\
\hline Total mastectomy & $1(2.32)$ \\
\hline None & $18(41.86)$ \\
\hline \multicolumn{2}{|l|}{ Recurrence } \\
\hline No & $31(72.09)$ \\
\hline Yes & $12(27.90)$ \\
\hline
\end{tabular}



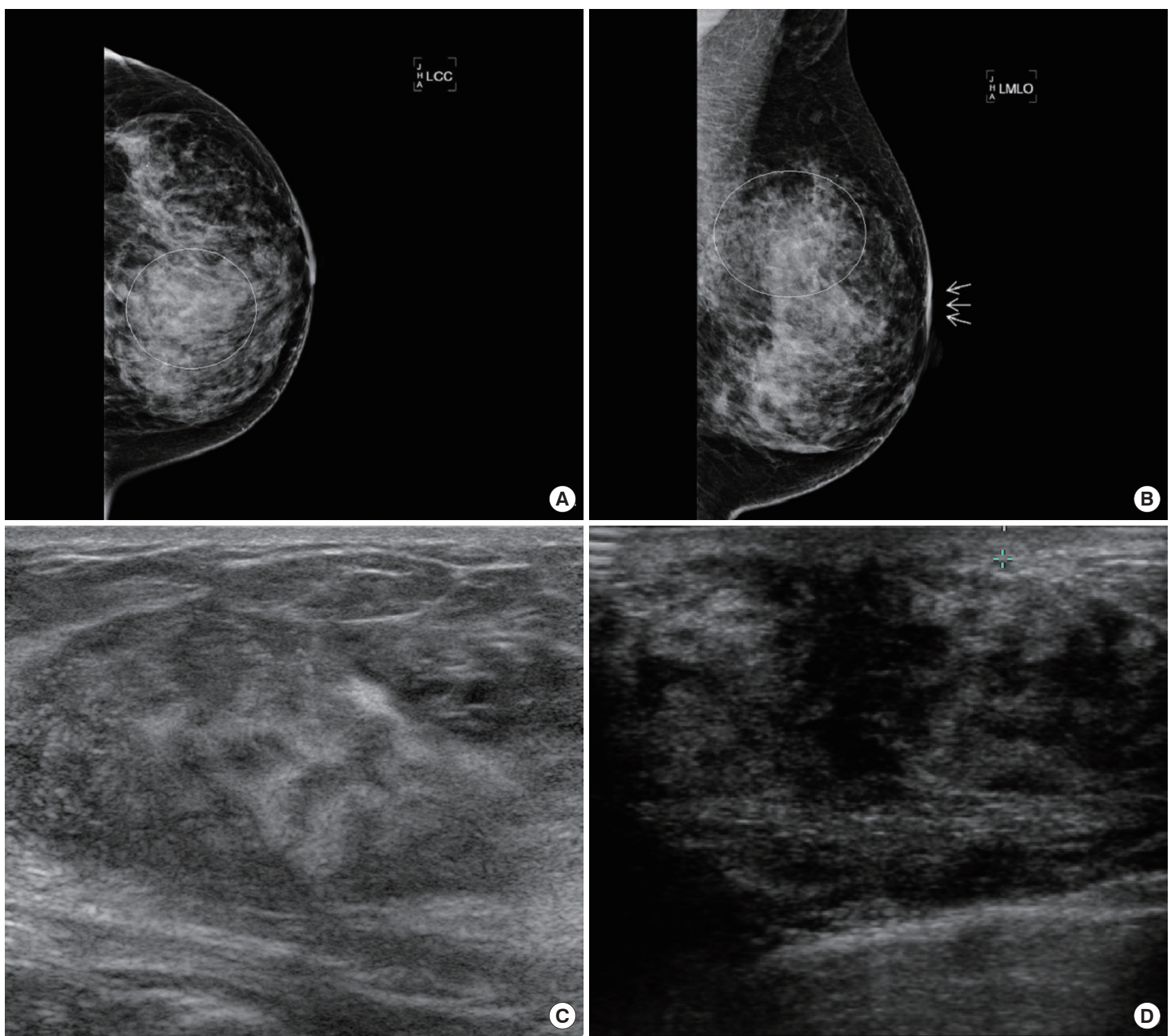

Figure 1. A 47-year-old woman with idiopathic granulomatous mastitis. (A, B) Mammograms showing a focal asymmetric hyperdense lesion (circle) and focal skin thickening (arrows). (C, D) Sonograms showing an ill-defined, heterogeneous, hypoechoic lesion with associated skin thickening.

iatrogenic Cushing's syndrome. Although the steroids were administered for more than 6 weeks, nine patients did not respond to treatment. Eighteen of the 43 study patients (41.86\%) underwent only nonsurgical treatment. In 24 patients (55.81\%), partial mastectomy was performed for lesion removal. Only one patient underwent total mastectomy owing to multiple lesions and steroid resistance.

Twelve patients (27.90\%) developed recurrence, but there was no evidence of recurrence in the other 31 patients. Eight (66.6\%) patients developed ipsilateral recurrence and four contralateral recurrence (Table 1).
Mammography was performed in 28 patients over the age of 30 years. Of these, 15 (53.57\%) showed focal asymmetric hyperdense lesions (Figure 1A) and seven (25.00\%) showed ill-defined masses (Figure $2 \mathrm{~A})$. In five patients (17.85\%), mammography revealed no specific findings other than a dense breast (Figure 1B). Two patients (7.14\%) had focal skin thickening (Figure 2B) and six patients (21.42\%) exhibited axillary lymph node enlargement (Table 2).

The most common ultrasonographic finding (22 patients, 51.16\%) was an ill-defined, heterogeneous, hypoechoic lesion (Figure 1C, D). Sixteen of the study patients (37.20\%) had an irregular hypoechoic 

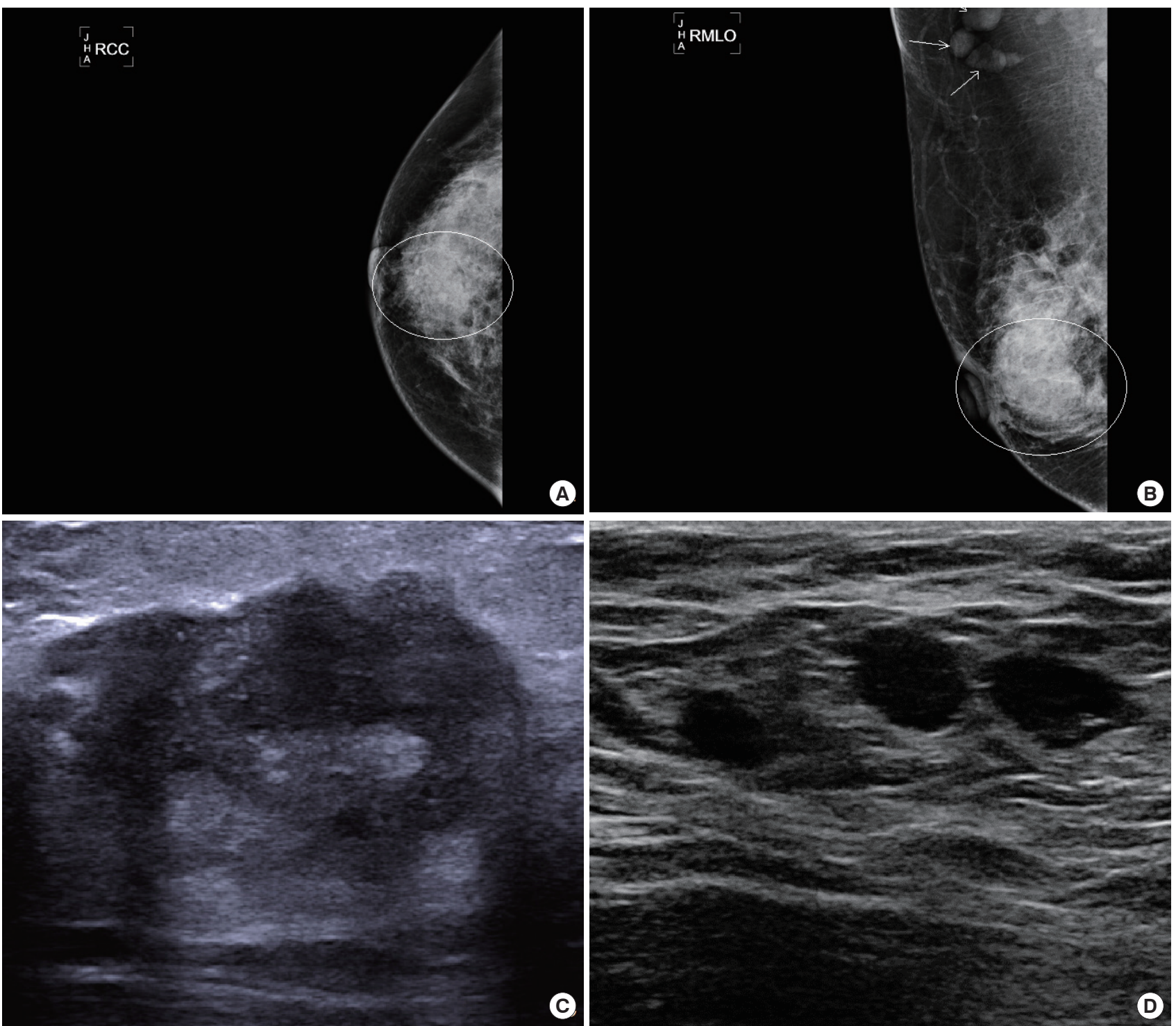

Figure 2. A 35-year-old woman with idiopathic granulomatous mastitis with palpable mass and axillary adenopathy by clinical examination. (A, B) Mammograms showing ill-defined mass density (circle) and suspected pathologic axillary lymph node enlargement (arrows). Sonograms showing an irregular hypoechoic mass (C) and associated hypoechoic abnormal lymph nodes (D).

Table 2. Mammographic findings of idiopathic granulomatous mastitis $(n=28)$

\begin{tabular}{lc}
\hline Finding & No. (\%) \\
\hline Hyperdense focal asymmetry & $15(53.57)$ \\
Irregular mass & $7(25.00)$ \\
Extremely dense breast & $5(17.85)$ \\
Nodular density & $1(3.57)$ \\
Axillary lymph node changes & $6(21.42)$ \\
Overlying skin thickening & $2(7.14)$ \\
\hline
\end{tabular}

mass (Figure 2C). In three patients, a tubular, hypoechoic lesion was observed; in two patients, only parenchymal distortion was observed.
In nine patients (20.93\%), the perilesional fat tissue showed increased echogenicity and in two patients (4.65\%), accompanying duct dilatation was observed. In three patients (6.97\%), skin thickening was evident (Figure 2D). Upon ultrasonography, 24 patients (55.81\%) did not show axillary lymph node enlargement, 16 patients (37.20\%) had enlarged benign lymph nodes and three patients (6.97\%) had an enlarged lymph node suspicious of malignancy.

According to the Breast Imaging-Reporting and Data System (BIRADS), 13 patients (30.23\%) demonstrated category 3 lesions and the remaining 30 (69.77\%) demonstrated category > 4A lesions; that is, 
these cases could be considered as suspected malignancy (Table 3).

\section{DISCUSSION}

Although several cases of IGM of unknown origin and with nonspecific clinical features have been reported, its diagnosis and treatment have remained controversial. The underlying pathogenesis of

Table 3. Ultrasonographic findings of idiopathic granulomatous mastitis $(\mathrm{n}=43)$

\begin{tabular}{lc}
\hline Finding & No. (\%) \\
\hline Finding of lesions & \\
Irregular hypoechoic lesion & $22(51.16)$ \\
Irregular hypoechoic mass & $16(37.20)$ \\
Tubular shaped hypoechoic lesion & $3(6.97)$ \\
Parenchymal distortion & $2(4.65)$ \\
Accompanied finding & \\
Increased echogenicity of parenchyma & $9(20.93)$ \\
Skin thickening & $3(6.97)$ \\
Duct dilatation & $2(4.65)$ \\
Axillary lymphadenopathy & \\
None & $24(55.81)$ \\
Benign & $16(37.20)$ \\
Suspected malignancy & $3(6.97)$ \\
No accompanied & $29(67.44)$ \\
BI-RADS & \\
3 & $13(30.23)$ \\
$4 A$ & $19(44.18)$ \\
$4 B$ & $8(18.60)$ \\
5 & $3(6.97)$ \\
\hline
\end{tabular}

BI-RADS = Breast Imaging-Reporting and Data System .
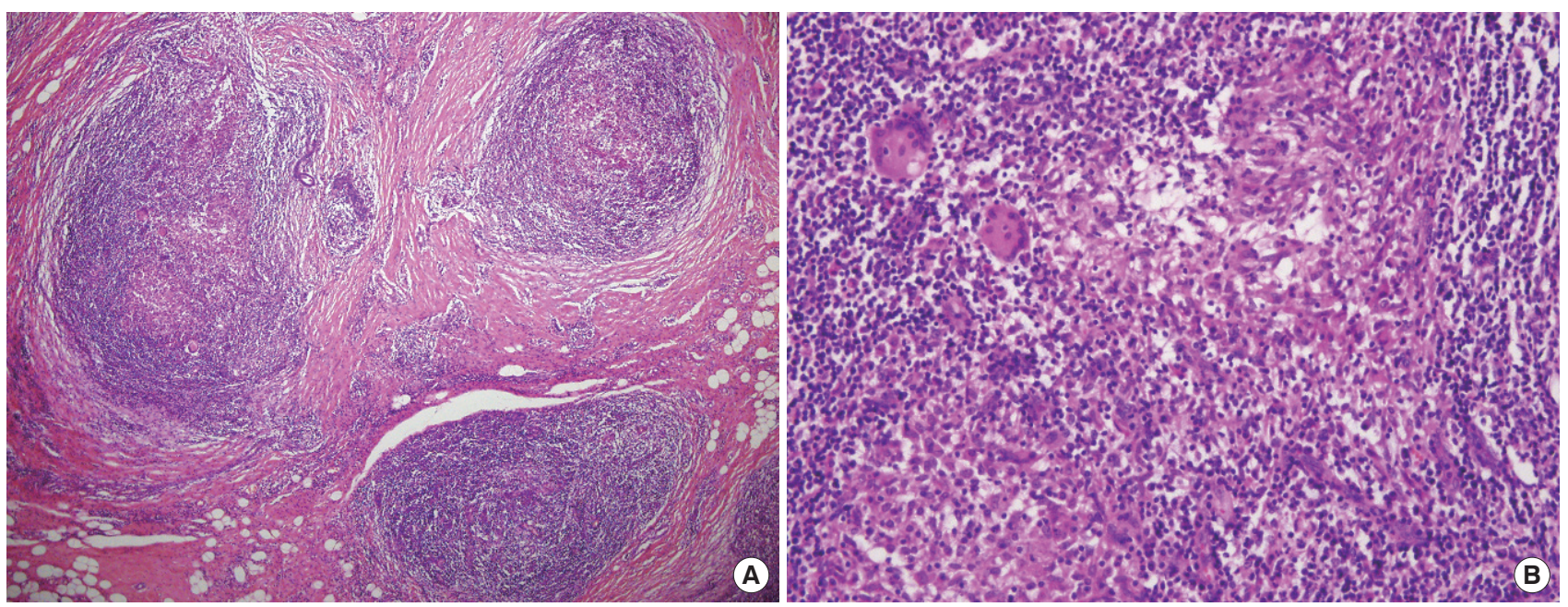

Figure 3. Pathophysiologic findings of idiopathic granulomatous mastitis. (A) Low-power view showing noncaseating granulomatous inflammation (H\&E stain, $\times 40)$. (B) High-power view showing epithelioid histiocytes, multinucleated giant cells, and lymphocyte infiltration (H\&E stain, $\times 100)$. 
tinction from breast cancer or granulomas of other origins on the basis of typical pathologic findings [1-4]. In our study, specimens were collected using FNA, CNB or both. The procedure was determined according to the discretion of the practitioner and the time of diagnosis. Therefore, a difference in diagnosis rates according to each method can be anticipated. Thirty patients (69.77\%) were found to have BI-RADS category $>4 \mathrm{~A}$ lesions, as assessed via mammography and ultrasonography. This is in accordance with the finding of previous studies showing that IGM could not be diagnosed on the basis of radiologic findings alone.

The pathological features of IGM include chronic granulomatous inflammation without necrosis (Figure 3A). Granulomas typically contain lymphocytes, plasma cells, epithelioid histiocytes, multinucleated giant cells, and rarely neutrophils (Figure 3B); granulomatous lesions can be observed in breast lobules or terminal ducts in any breast quadrant except subareolar region [3]. Infectious causes of granuloma of the breast include corynebacterial infection, tuberculosis, cat-scratch disease, and fungal infection. Noninfectious causes include sarcoidosis, Wegener's granulomatosis, giant cell arteritis, polyarteritis nodosa, ductal ectasia, foreign body reaction, and granulomatous reactions due to breast cancer [8-10]. Infectious causes should be diagnosed differentially, taking into account the corticosteroid usage. Tuberculosis arising from the breast is a rare disease, but the incidence of breast tuberculosis as a complication of acquired immunodeficiency syndrome is increasing worldwide [11]. Moreover, when women of childbearing age complain of a palpable breast mass in a geographic area where tuberculosis is endemic (such as Korea), the possibility of breast tuberculosis should not be overlooked. Breast tuberculosis and other infectious causes can be excluded via serologic and pathologic testing, for example, by using bacterial and fungal cultures and specific stain-based methods [12-14].

There are still no diagnostic criteria or treatment guidelines for IGM. Before the 1980s, complete resection of the lesion was the only treatment applied [9]. Since DeHertogh et al. [15] first described the usefulness of corticosteroid treatment, it has been considered a therapeutic option, and although effective, high doses cause side effects such as glucose intolerance or Cushing's syndrome. In recent studies, low-dose steroids have been recommended as first-line therapy $[6,11]$, and although extensive surgical resection is still recommended by some physicians, corticosteroid administration may be helpful in avoiding the need for surgery. Initially, as a result of a study performed by DeHertogh et al. [15] in the 1980s, high-dose steroid treatment was routinely administered, the well-known side effects of steroids were noted and lower doses were recommended [2,6-8,13]. At our center, we administered steroids at an initial dose of $0.4 \mathrm{mg} / \mathrm{kg} /$ day. All patients underwent steroid treatment without any side effects, and 18 of 43 steroid-treated patients did not require surgery. The steroid dose was tapered for 1 to 28 weeks depending on symptom improvement or changes in clinical features. If recurrence occurs after mastectomy due to a poor response to steroids, reoperation or high-dose steroid treatment may be effective in cases of delayed relapse or complete recovery [11]. In addition, in one study, methotrexate was found to be a favorable option for patients with recurrence or steroid-resistance [16].

IGM is rare and is sometimes misdiagnosed as simple mastitis or breast cancer. However, when diagnosed timely through radiologic and histologic examination, and on the basis of low-dose corticosteroid usage, clinical outcomes can be improved without the need for surgical treatment. Delayed diagnosis and treatment could result in unnecessarily prolonged pain, abscess or fistula formation, and cosmetic impairments. In view of the fact that almost all patients with IGM are young and socially active women, this is not only a personal problem, but also a socioeconomic one. In conclusion, we recommend that studies be undertaken to determine the etiology of IGM and establish rapid and appropriate diagnostic and therapeutic protocols.

\section{CONFLICT OF INTEREST}

The authors declare that they have no competing interests.

\section{REFERENCES}

1. Kessler E, Wolloch Y. Granulomatous mastitis: a lesion clinically simulating carcinoma. Am J Clin Pathol 1972;58:642-6.

2. Fletcher A, Magrath IM, Riddell RH, Talbot IC. Granulomatous mastitis: a report of seven cases. J Clin Pathol 1982;35:941-5.

3. Jorgensen MB, Nielsen DM. Diagnosis and treatment of granulomatous mastitis. Am J Med 1992;93:97-101.

4. Donn W, Rebbeck P, Wilson C, Gilks CB. Idiopathic granulomatous mastitis: a report of three cases and review of the literature. Arch Pathol Lab Med 1994;118:822-5. 
5. Hovanessian Larsen LJ, Peyvandi B, Klipfel N, Grant E, Iyengar G. Granulomatous lobular mastitis: imaging, diagnosis, and treatment. AJR Am J Roentgenol 2009;193:574-81.

6. Lin CH, Hsu CW, Tsao TY, Chou J. Idiopathic granulomatous mastitis associated with risperidone-induced hyperprolactinemia. Diagn Pathol 2012;7:2.

7. Rowe PH. Granulomatous mastitis associated with a pituitary prolactinoma. Br J Clin Pract 1984;38:32-4.

8. Goldberg J, Baute L, Storey L, Park P. Granulomatous mastitis in pregnancy. Obstet Gynecol 2000;96(5 Pt 2):813-5.

9. Imoto S, Kitaya T, Kodama T, Hasebe T, Mukai K. Idiopathic granulomatous mastitis: case report and review of the literature. Jpn J Clin Oncol 1997;27:274-7.

10. Taylor GB, Paviour SD, Musaad S, Jones WO, Holland DJ. A clinicopathological review of 34 cases of inflammatory breast disease showing an association between corynebacteria infection and granulomatous mastitis. Pathology 2003;35:109-19.
11. Bakaris S, Yuksel M, Ciragil P, Guven MA, Ezberci F, Bulbuloglu E. Granulomatous mastitis including breast tuberculosis and idiopathic lobular granulomatous mastitis. Can J Surg 2006;49:427-30.

12 Akcan A, Akyildiz H, Deneme MA, Akgun H, Aritas Y. Granulomatous lobular mastitis: a complex diagnostic and therapeutic problem. World J Surg 2006;30:1403-9.

13. Osborne BM. Granulomatous mastitis caused by histoplasma and mimicking inflammatory breast carcinoma. Hum Pathol 1989;20: 47-52.

14. Davies JD, Burton PA. Postpartum lobular granulomatous mastitis. J Clin Pathol 1983;36:363.

15. DeHertogh DA, Rossof AH, Harris AA, Economou SG. Prednisone management of granulomatous mastitis. N Engl J Med 1980; 303:799-800

16. Kim J, Tymms KE, Buckingham JM. Methotrexate in the management of granulomatous mastitis. ANZ J Surg 2003;73:247-9. 